\title{
$\mathrm{Pt}_{0.01} \mathrm{Fe}_{0.05}-\mathrm{g}-\mathrm{C}_{3} \mathrm{~N}_{4}$ 催化剂高效光热催化二氧化碳还原

\author{
王瑞兆 $a, b$ 邹云杰 $a, b$ 洪易 $a, b$ 徐铭楷 $a, b$ 凌岗*,a,b \\ ( ${ }^{a}$ 同济大学 污染控制与资源化研究国家重点实验室 上海 200092) \\ ( $b$ 福州大学 能源与环境光催化国家重点实验室 福州 350116)
}

\begin{abstract}
摘要 光热催化是一种高效利用太阳光, 将二氧化碳转化为高价值产物的方法. 本工作以石墨相氮化碳为载体, 通过 水热-浸渍两步法制备了负载铂、铁氧化物的石墨相氮化碳催化剂. 该催化剂具备优异的光热转换性能, 可实现 7.36 $\mathrm{mmol} \cdot \mathrm{h}^{-1} \cdot \mathrm{gcat}^{-1}$ 的二氧化碳还原活性和 $97 \%$ 的一氧化碳选择性. 使用 X 射线晶体衍射(XRD)、配备能量色散 X 射线谱 (EDS)的球差校正扫描透射电子显微镜(Cs-S/TEM)、X 射线光电子能谱(XPS)、紫外可见漫反射光谱(DRS)等表征手段 从催化剂物相、微观结构、表面状态、光学性能等方面对催化剂进行了表征. 结果显示, 催化剂能吸收全谱太阳光, 且 具备较高的载流子分离效率. 基于原位傅里叶变换红外光谱(DRIFTS)表征结果, 提出了二氧化碳在催化剂表面的可能 的反应机理, 并对铂在铁氧化物表面的氢溢流效应进行了表征. 结果表明二氧化碳和氢气分别在铁氧化物、铂位点被 活化, 参与催化反应. 本工作对后续光热二氧化碳还原催化剂的设计、合成与机理研究具有一定的参考作用. 关键词 石墨相氮化碳; 光热催化; 二氧化碳还原; 原位傅里叶变换红外光谱
\end{abstract}

\section{High-performance $\mathrm{Pt}_{0.01} \mathrm{Fe}_{0.05}-\mathrm{g}-\mathrm{C}_{3} \mathrm{~N}_{4}$ Catalyst for Photothermal Catalytic $\mathrm{CO}_{2}$ Reduction}

\author{
Ruizhao Wang ${ }^{a, b}$ Yunjie Zou ${ }^{a, b}$ Sheng Hong ${ }^{a, b}$ Mingkai Xu ${ }^{a, b}$ Lan Ling ${ }^{*, a, b}$ \\ ( ${ }^{a}$ State Key Laboratory of Pollution Control and Resource Reuse, Tongji University, Shanghai 200092, China) \\ ( ${ }^{b}$ State Key Laboratory of Photocatalysis on Energy and Environment, Fuzhou University, Fuzhou 350116, China)
}

\begin{abstract}
Converting $\mathrm{CO}_{2}$ into value-added compounds via photothermal catalysis is a promising strategy to reduce $\mathrm{CO}_{2}$ emission and might be a sustainable alternative to traditional fossil fuels. Here, we report nano-structured a $\mathrm{Pt}_{0.01} \mathrm{Fe}_{0.05}-\mathrm{g}-\mathrm{C}_{3} \mathrm{~N}_{4}$ hybrid catalyst synthesized via hydrothermal-method and further reduced under reaction condition for the reverse water gas shift (RWGS) reaction. Taking advantage of the photo-thermal effect caused by the near-infrared (NIR) and visible light responsive, the hybrid catalyst produces a remarkable activity $\left(7.36 \mathrm{mmol} \bullet \mathrm{h}^{-1} \cdot \mathrm{g}_{\mathrm{cat}}{ }^{-1}\right)$ for $\mathrm{CO}_{2}$ reduction with $\mathrm{CO}$ selectivity $(97 \%)$ under $300 \mathrm{~W}$ Xe lamp irradiation and $\mathrm{CO}_{2} / \mathrm{H}_{2}(V / V, 1 / 1)$ feed gas. The apparent activation energy of reaction decreases from $238.59 \mathrm{~kJ} / \mathrm{mol}$ to $48.88 \mathrm{~kJ} / \mathrm{mol}$ calculated by Arrhenius formula. In order to comprehend the good catalytic activity of $\mathrm{Pt}_{0.01} \mathrm{Fe}_{0.05}-\mathrm{g}-\mathrm{C}_{3} \mathrm{~N}_{4}$ in the RWGS reaction, the catalyst is characterized with powder X-ray diffraction (XRD), spherical-aberration-corrected scanning transmission electron microscopy (Cs-S/TEM) integrated with X-ray energy dispersive spectroscopy (EDS), X-ray photoelectron spectroscopy (XPS), UV-vis-NIR diffuse reflectance spectroscopy (DRS), etc. for investigating the structural information, surface state, optical properties and so on. Results show that the presence of $\mathrm{FeO}_{x}$ and Pt exhibits strong absorption in a wide range from UV to NIR regions. Low photoluminescence (PL) intensity at about $460 \mathrm{~nm}$ shows the suppressed photogenerated carrier recombination process of $\mathrm{Pt}_{0.01} \mathrm{Fe}_{0.05}-\mathrm{g}_{-} \mathrm{C}_{3} \mathrm{~N}_{4}$ due to the heterojunction between $\mathrm{FeO}_{x}$ and $\mathrm{g}_{-} \mathrm{C}_{3} \mathrm{~N}_{4}$. Operando diffuse reflectance infrared Fourier transform spectroscopy (DRIFTS) under different reaction conditions is employed to investigate surface species and their evolution during the conversion of $\mathrm{CO}_{2}$ into $\mathrm{CO}$ and a broad IR absorption is observed due to hydrogen spillover from $\mathrm{Pt}$ to $\mathrm{Fe}_{3} \mathrm{O}_{4}$. Therefore, we propose a possible mechanism of photothermal catalytic $\mathrm{CO}_{2}$ reduction, involving separate activation of $\mathrm{CO}_{2}$ and $\mathrm{H}_{2}$ over $\mathrm{Fe}$ and $\mathrm{Pt}$ acitve sites. Our work present a high-performance $\mathrm{Pt}_{0.01} \mathrm{Fe}_{0.05}-\mathrm{g}-\mathrm{C}_{3} \mathrm{~N}_{4}$ catalyst for RWGS reaction and open a new vista of the design, synthesis and mechanism research of photothermal catalytic $\mathrm{CO}_{2}$ conversion in the future.
\end{abstract}

Keywords g- $\mathrm{C}_{3} \mathrm{~N}_{4}$; photothermal catalysis; $\mathrm{CO}_{2}$ reduction reaction; operando diffuse reflectance infrared Fourier transform spectroscopy

\footnotetext{
* E-mail: linglan@tongji.edu.cn

Received March 29, 2021; published May 29, 2021.

Supporting information for this article is available free of charge via the Internet at http://sioc-journal.cn.

Project supported by the National Natural Science Foundation of China (No. 21822607) and State Key Laboratory of Photocatalysis on Energy and Environment (No. SKLPEE-KF201701).

项目受国家自然科学基金优秀青年基金(No. 21822607)和能源与环境光催化国家重点实验室开放课题(No. SKLPEE-KF201701)资助.
} 


\section{1 引言}

随着工业技术的发展, 人们对能源的需求量与日俱 增. 化石燃料的开发利用导致了大量二氧化碳排放. 截 至 2020 年 5 月 30 日, 大气中二氧化碳的平均浓度已经 增高到了 $0.041647 \%$, 相比进入工业社会前的 $0.0172 \% \sim 0.03 \%$ 增加了 $0.01 \%$ 以上, 引发了严重的温室 效应 ${ }^{[1]}$. 然而, 根据相关研究机构预测, 全球对能源的 需求仍会持续提高, 预计 2040 年能源需求量相比现在 将增长 $30 \%$ 左右 ${ }^{[2]}$. 为了减少温室气体排放的同时缓解 化石能源的短缺, 开发新型的环境友好可再生能源刻不 容缓. 太阳能作为最常见的可再生能源, 是地球能量的 主要来源, 具有储量丰富、清洁无污染的特点 ${ }^{[3]}$. 通过光 催化, 将光能直接转化为含碳燃料中的化学能被认为是 一种可以同时解决温室效应与能源危机的方案 ${ }^{[4]}$.

光催化剂的光吸收性能在一定程度上决定其光催 化效率, 传统光催化通常使用半导体催化剂, 受限于半 导体的能带结构, 只能利用能量大于禁带宽度阈值的光 子 [2]. 对于大部分金属氧化物半导体而言, 这一阈值主 要分布于太阳光光谱的紫外区, 而紫外光光子仅占到达 地球表面的光子中不足 $2 \%$. 应对这一太阳光利用效 率低问题, 光热催化理论上可以吸收全波长太阳光谱范 围的光子, 其中能量高于禁带宽度的光子可被吸收并激 发价带电子产生光生载流子; 能量低于禁带宽度的光子 可诱导声子弛豫产生强烈热效应, 二者共同促进催化剂 表面化学反应的发生 ${ }^{[5-6]}$. 综上, 光热反应催化剂在催化 反应中同时起到提供反应所需温度和作为光催化活性 位点的双重作用. 因此, 高效的光热反应催化剂需要具 有光吸收与光响应性能: (1)在全太阳光谱范围存在较强 吸收, 提高反应位点局部温度, 驱动表面热化学反应; (2)高的电子-空穴产生效率与载流子分离效率, 促进表 面光化学反应的发生.

为了增强光热催化反应效率, 以往的研究通过构建 各种复合结构, 实现了优异的光热催化二氧化碳还原性 能. Hoch 等 ${ }^{[7]}$ 设计了 $\operatorname{In}_{2} \mathrm{O}_{3-x}(\mathrm{OH})_{y}$ 与垂直排列硅纳米线 $(\mathrm{SiNW})$ 的杂交结构, 以宽带隙的 $\mathrm{In}_{2} \mathrm{O}_{3-x}(\mathrm{OH})_{y}$ 作为二氧 化碳反应位点, $\mathrm{SiNW}$ 将能量低于 $\operatorname{In}_{2} \mathrm{O}_{3-x}(\mathrm{OH})_{y}$ 带隙的光 能转化为热, 获得了 $0.022 \mathrm{mmol} \cdot \mathrm{h}^{-1} \cdot \mathrm{g}_{\mathrm{cat}}{ }^{-1}$ 的 $\mathrm{CO}$ 产生速 率; $\mathrm{Li}$ 等 ${ }^{[8]}$ 制备了 $\mathrm{Cu}$ 原子负载的 $\mathrm{Pd} / \mathrm{H}_{y} \mathrm{WO}_{3-x}$ 纳米线, 藉 由乌青铜在全太阳光谱波段的强吸收, 获得了 0.073 $\mathrm{mmol} \cdot \mathrm{h}^{-1} \cdot \mathrm{g}_{\mathrm{cat}}{ }^{-1}$ 的 $\mathrm{CO}$ 活性; $\mathrm{Jia}$ 等 ${ }^{[9]}$ 将 $\mathrm{Pd}$ 纳米晶体负载 于 $\mathrm{Nb}_{2} \mathrm{O}_{5}$ 纳米棒, 利用 $\mathrm{Pd}$ 纳米晶体带内与带间跃迁产 生的在可见-近红外光 $(400 \sim 2400 \mathrm{~nm}$ )吸收进行光热转 换, 同时体系中的 $\mathrm{Pd}$ 和 $\mathrm{Nb}_{2} \mathrm{O}_{5}$ 分别作为氢气与二氧化 碳活化位点参与反应, 获得了 $4.9 \mathrm{mmol} \cdot \mathrm{h}^{-1} \cdot \mathrm{g}_{\mathrm{cat}}{ }^{-1}$ 的 $\mathrm{CO}$ 活性. 然而, 高 $\mathrm{CO}$ 选择性与催化活性往往不可兼得, 研究高效、高 $\mathrm{CO}$ 选择性的催化剂一直是光热催化领域 的目标.

石墨相氮化碳 $\left(\mathrm{g}-\mathrm{C}_{3} \mathrm{~N}_{4}\right)$ 因其合适的能带结构、化学
稳定性、热稳定性引起了研究者们的注意 ${ }^{[10]}$. 自 2009 年 Wang 等将 $\mathrm{g}-\mathrm{C}_{3} \mathrm{~N}_{4}$ 应用于无牺牲剂光解水反应以来, 零维、一维、二维、三维结构的 $\mathrm{g}-\mathrm{C}_{3} \mathrm{~N}_{4}$ 陆续被合成, 并 应用于光解水、光降解染料、二氧化碳还原等太阳能转 化领域 ${ }^{[11-12]}$. 尽管如此, $\mathrm{g}-\mathrm{C}_{3} \mathrm{~N}_{4}$ 光生载流子复合率高、电 导率低、460 nm 以上波长光利用率低等众多缺点是其在 更多催化体系中应用的挑战因素. 为了克服以上不足, Huang 等[13]使用过氧化氢水热法合成了氧掺杂的 $\mathrm{g}-\mathrm{C}_{3} \mathrm{~N}_{4}$, 扩展了 $\mathrm{g}-\mathrm{C}_{3} \mathrm{~N}_{4}$ 光吸收范围; Dong 等[14]将 $\mathrm{La}$ 针 定于 $\mathrm{g}-\mathrm{C}_{3} \mathrm{~N}_{4}$, 增强其层间电子传输效率, 提高了二氧化 碳还原活性; $\mathrm{Fu}$ 等 ${ }^{[15]}$ 于 $\mathrm{g}-\mathrm{C}_{3} \mathrm{~N}_{4}$ 与 $\mathrm{WO}_{3}$ 界面构筑异质结, 降低了光生载流子复合率，增强了对亚甲基蓝和碱性品 红的光降解能力.

基于以上原因, 本工作通过在 $\mathrm{g}-\mathrm{C}_{3} \mathrm{~N}_{4}$ 负载铁氧化 物和铂, 在 $\mathrm{g}-\mathrm{C}_{3} \mathrm{~N}_{4}$ 表面构筑异质结构降低载流子复合 率、拓展光吸收范围, 负载的铂和铁氧化物还可以分别 作为氢气和二氧化碳的活化位点, 进行二氧化碳光热还 原反应. 本工作通过控制元素组成、结构、空间分布合 成催化剂, 并对催化剂的结构与性能进行考察, 从催化 剂结构、光学性能、反应机理等方面对二氧化碳光热还 原反应机制进行了讨论, 为后续二氧化碳光热还原反应 催化剂的设计与开发提供一定参考.

\section{2 结果与讨论}

\section{1 物相分析}

不同 $\mathrm{Fe} 、 \mathrm{Pt}$ 负载量的 $\mathrm{Pt}_{x} \mathrm{Fe}_{y}-\mathrm{g}-\mathrm{C}_{3} \mathrm{~N}_{4}$ 催化剂通过水 热法与浸渍法两步合成, 并在光照至 $300{ }^{\circ} \mathrm{C} 、 \mathrm{CO}_{2} / \mathrm{H}_{2}$ $(V / V, 1 / 1)$ 、总流量 $40 \mathrm{~mL} / \mathrm{min}$ 条件下进行还原. 为简化 描述，本文将铁负载量为 $5 \%(w) 、 0.5 \%(w) 、 0(w)$ 简记 为 $5 \mathrm{Fe} 、 0.5 \mathrm{Fe} 、 0 \mathrm{Fe}$; 铂负载量为 $1 \%(w) 、 0(w)$ 简记为 $1 \mathrm{Pt} 、 0 \mathrm{Pt}$; 还原前、后分别记为 $\mathrm{BR}$ (before reduction)、 $\mathrm{AR}$ (after reduction), 如未还原的铁负载量为 5\%(w)、铂 负载量为 $1 \%(w)$ 的催化剂记作 $5 \mathrm{Fe} 1 \mathrm{Pt}$ BR.

为探究催化剂的物相结构, 我们对系列催化剂进行 了 $\mathrm{X}$ 射线晶体衍射(XRD)表征, 结果如图 1 所示. 图中 为三组含铁量为 $5 \%(w), \mathrm{Pt}$ 含量为 $1 \%(w)$ 或 $0(w)$ 的样品 的 XRD 谱图以及 $\mathrm{Fe}_{2} \mathrm{O}_{3}$ 和 $\mathrm{Fe}_{3} \mathrm{O}_{4}$ 的标准 $\mathrm{PDF}$ 卡片 (PDF\#33-0664、PDF\#04-0802). 由结果可知, 5Fe1Pt BR 与 $5 \mathrm{Fe} 0 \mathrm{Pt}$ 催化剂的 XRD 衍射图谱均由分别归属于 $(002)$ 与(100)衍射面, $2 \theta$ 为 $27.8^{\circ}$ 和 $13.3^{\circ}$ 的 $\mathrm{g}-\mathrm{C}_{3} \mathrm{~N}_{4}$ 特征衍射峰 (图 S1) 与 $2 \theta$ 为 $24.1^{\circ} 、 33.2^{\circ} 、 40.9^{\circ} 、 49.5^{\circ} 、 54.1^{\circ} 、 57.6^{\circ}$ 、 $62.4^{\circ} 、 64.0^{\circ} 、 71.9^{\circ}$, 分别归属于赤铁矿 $(012) 、(104) 、$ (110)、(113)、(024)、(116)、(018)、(214)、(300)、(1010) 衍射面的特征衍射峰组成. $5 \mathrm{Fe} 1 \mathrm{Pt} \mathrm{BR}$ 与 $5 \mathrm{Fe} 0 \mathrm{Pt}$ 催化剂 $\mathrm{XRD}$ 谱图之间存在较高相似性, 均只包含 $\mathrm{Fe}_{2} \mathrm{O}_{3}$ 与 $\mathrm{g}-\mathrm{C}_{3} \mathrm{~N}_{4}$ 特征衍射峰, 表明浸渍合成前后催化剂的结构并 未发生改变. 而还原后的 $5 \mathrm{Fe} 1 \mathrm{Pt}$ 催化剂的 XRD 谱图除 了 $\mathrm{g}-\mathrm{C}_{3} \mathrm{~N}_{4}$ 特征衍射峰, 由 $2 \theta$ 为 $18.3^{\circ} 、 30.1^{\circ} 、 35.4^{\circ} 、 37.1^{\circ}$ 、 
$43.1^{\circ} 、 53.4^{\circ} 、 56.9^{\circ} 、 62.5^{\circ} 、 73.9^{\circ}$, 分别归属于(111)、 (220)、(311)、(222)、(400)、(422)、(511)、(440)磁铁矿 衍射面和较弱的归属于 (111) 衍射面, $2 \theta$ 为 $39.8^{\circ}$ 的金属 铂特征衍射峰(PDF\#04-0802) (图 S2)组成. 还原前后 $5 \mathrm{Fe} 1 \mathrm{Pt}$ 催化剂的 XRD 谱图对应晶相不同, 这表明在原 位还原过程中, 催化剂发生了晶相转变, 催化剂中铁元 素的存在形式由赤铁矿型 $\mathrm{Fe}_{2} \mathrm{O}_{3}$ 转变成了磁铁矿型 $\mathrm{Fe}_{3} \mathrm{O}_{4}$, 并伴随部分单质铂产生.

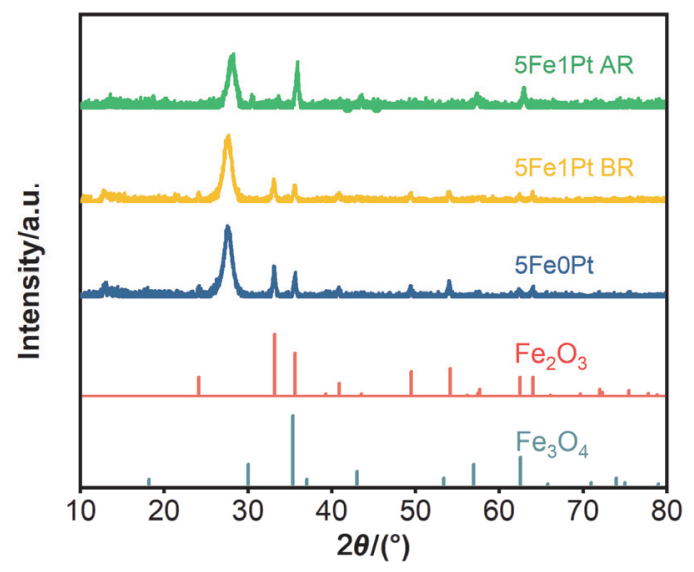

图 $15 \mathrm{Fe} 1 \mathrm{Pt} \mathrm{AR}, 5 \mathrm{Fe} 1 \mathrm{Pt} \mathrm{BR}$ 和 $5 \mathrm{Fe} 0 \mathrm{Pt}$ 样品的 XRD 谱图

Figure 1 XRD patterns of $5 \mathrm{Fe} 1 \mathrm{Pt} \mathrm{AR}, 5 \mathrm{Fe} 1 \mathrm{Pt} \mathrm{BR}$ and $5 \mathrm{Fe} 0 \mathrm{Pt}$
为了研究还原过程中催化剂表面元素价态的变化, 我们对 $5 \mathrm{Fe} 1 \mathrm{Pt} \mathrm{BR} 、 5 \mathrm{Fe} 1 \mathrm{Pt} \mathrm{AR}$ 两组催化剂进行了 $\mathrm{X}$ 射 线光电子能谱(XPS)表征. 样品的 XPS 全谱图如图 2a 所 示, 可以看出 $5 \mathrm{Fe} 1 \mathrm{Pt}$ AR 催化剂的 XPS 全谱图中存在归 属于 $\mathrm{Fe} 2 \mathrm{p}$ 和 $\mathrm{Pt} 4 \mathrm{f}$ 的特征光电子信号. 这说明催化剂 $\mathrm{Pt}$ 和 $\mathrm{Fe}$ 的成功负载. 为了精确识别各物种的存在形式, 对 $\mathrm{Fe} 2 \mathrm{p}_{3 / 2}$ 与 $\mathrm{Pt} 4 \mathrm{f}$ 的精细 XPS 谱图进行了分析. 5Fe1Pt BR 催化剂 $\mathrm{Fe} 2 \mathrm{p}_{3 / 2}$ 精细谱中结合能 709.8、710.7、711.4、 $712.3 \mathrm{eV}$ 处的峰, 归属于 $\alpha-\mathrm{Fe}_{2} \mathrm{O}_{3}$ 中 $\mathrm{Fe}^{3+}$ 的多重谱线; $5 \mathrm{Fe} 1 \mathrm{Pt}$ AR 催化剂 Fe 2 $\mathrm{p}_{3 / 2}$ 精细谱中出现结合能 710.2、 $711.3 、 712.4 、 713.6 \mathrm{eV}$ 与 708.3、709.3、710.4 eV 的两 组峰则分别归属于 $\mathrm{Fe}_{3} \mathrm{O}_{4}$ 中的 $\mathrm{Fe}^{3+} 、 \mathrm{Fe}^{2+}$ 的多重谱线 ${ }^{[16]}$. 以上结果表明 $5 \mathrm{Fe} 1 \mathrm{Pt} \mathrm{BR}$ 与 $5 \mathrm{Fe} 1 \mathrm{Pt} \mathrm{AR}$ 催化剂中铁元素 分别以 $\mathrm{Fe}_{2} \mathrm{O}_{3}$ 与 $\mathrm{Fe}_{3} \mathrm{O}_{4}$ 的形态存在, 再次证明了原位还 原过程中铁元素的价态改变. 5Fe1Pt BR 催化剂 Pt 4f 精 细谱中结合能为 76.4、73.0 eV 两个位置的峰分别归属 于 $\mathrm{Pt}^{2+}$ 的 $4 \mathrm{f}_{5 / 2}$ 与 $4 \mathrm{f}_{7 / 2}$ 的特征峰 ${ }^{[17]}$. 还原后样品 $\mathrm{Pt} 4 \mathrm{f}$ 精 细谱中在较低结合能处出现了结合能为 74.4、71.1 eV 的一组新峰, 这一组新峰结合能与 $\mathrm{Pt}^{0} 4 \mathrm{f}_{5 / 2}$ 与 $4 \mathrm{f}_{7 / 2}$ 特征 峰一致，表明还原过程中产生了 $\mathrm{Pt}^{0}$ 物种 ${ }^{[18]}$. 同时, $5 \mathrm{Fe} 0 \mathrm{Pt}$ 与 $5 \mathrm{Fe} 1 \mathrm{Pt} \mathrm{AR} \mathrm{N} 1 \mathrm{~s}$ 精细谱的高度相似性说明了 $g-\mathrm{C}_{3} \mathrm{~N}_{4}$ 载体在合成、反应条件下的稳定性.
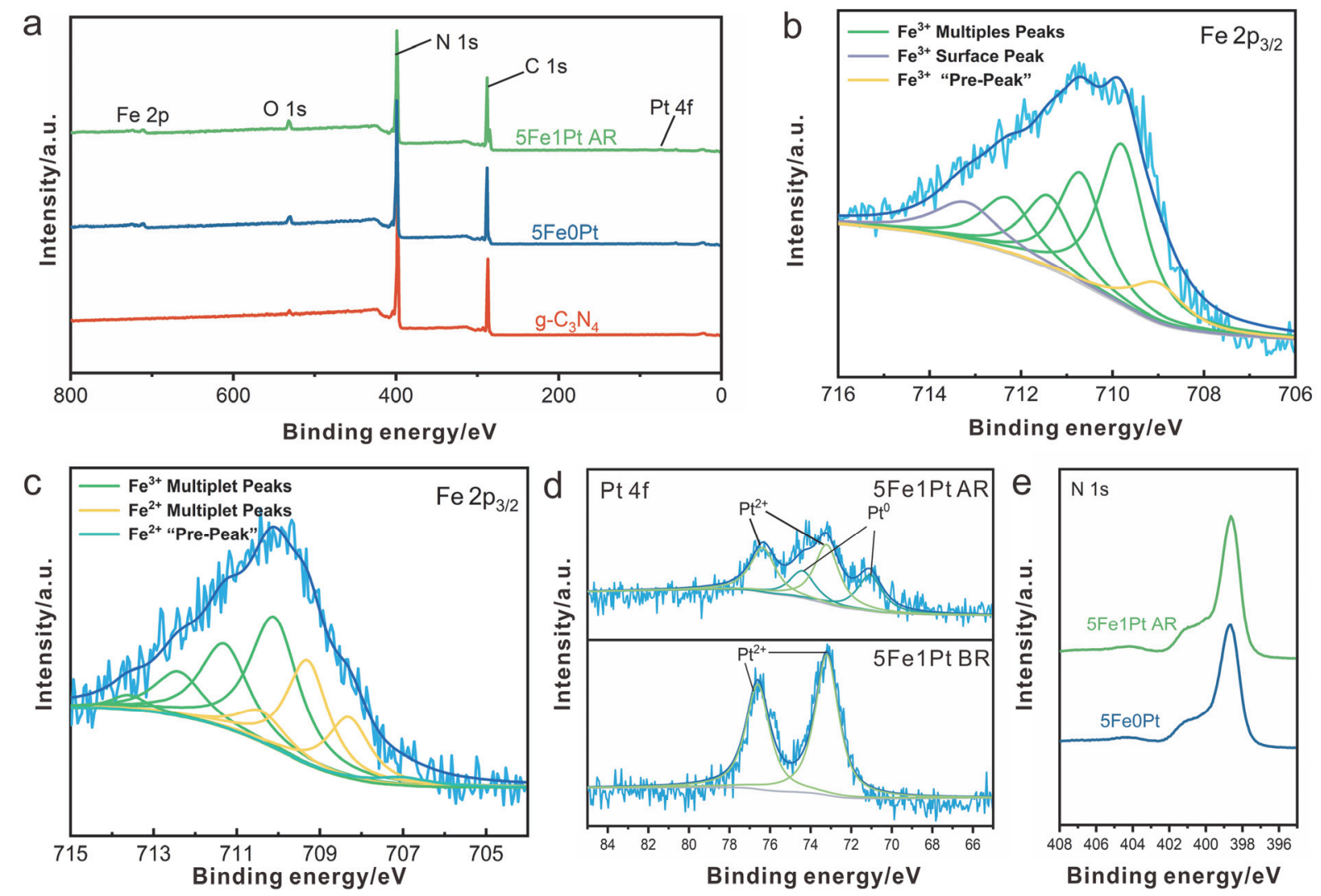

图 $25 \mathrm{Fe} 1 \mathrm{Pt} \mathrm{AR}, 5 \mathrm{Fe} 0 \mathrm{Pt}$ 和 $\mathrm{g}-\mathrm{C}_{3} \mathrm{~N}_{4}$ 的 XPS 总谱(a), $5 \mathrm{Fe} 1 \mathrm{Pt} \mathrm{BR}(\mathrm{b})$ 和 $5 \mathrm{Fe} 1 \mathrm{Pt} \mathrm{AR}(\mathrm{c})$ 的高自旋 $\mathrm{Fe} 2 \mathrm{p}$ XPS 谱图, $5 \mathrm{Fe} 1 \mathrm{Pt} \mathrm{BR}$ 和 $5 \mathrm{Fe} 1 \mathrm{Pt} \mathrm{AR}$ 的 Pt $4 \mathrm{f}$ XPS 谱图(d), $5 \mathrm{Fe} 0 \mathrm{Pt}$ 与 $5 \mathrm{Fe} 1 \mathrm{Pt} \mathrm{AR}$ 的 $\mathrm{N} 1 \mathrm{~s}$ XPS 谱图(e)

Figure 2 Full XPS pattern of $5 \mathrm{Fe} 1 \mathrm{Pt}$ AR, and $5 \mathrm{Fe} 0 \mathrm{Pt}$, g- $\mathrm{C}_{3} \mathrm{~N}_{4}$ (a), XPS pattern of high-spin Fe $2 \mathrm{p}$ for $5 \mathrm{Fe} 1 \mathrm{Pt} \mathrm{BR}(\mathrm{b})$ and $5 \mathrm{Fe} 1 \mathrm{Pt} \mathrm{AR}(\mathrm{c}), \mathrm{Pt} 4 \mathrm{f}$ for $5 \mathrm{Fe} 1 \mathrm{Pt}$ $\mathrm{BR}$ and $5 \mathrm{Fe} 1 \mathrm{Pt} \mathrm{AR}(\mathrm{d}), \mathrm{N} 1 \mathrm{~s}$ for $5 \mathrm{Fe} 0 \mathrm{Pt}$ and $5 \mathrm{Fe} 1 \mathrm{Pt} \mathrm{AR}(\mathrm{e})$ 
为表征催化剂的微观结构, 我们使用球差校正扫描 透射电子显微镜(Cs-S/TEM) 对催化剂微观结构进行了 表征. 高分辨透射电子显微镜像(high resolution transmission electron microscopy, HRTEM)结果如图 3a 所示, $5 \mathrm{Fe} 1 \mathrm{Pt} \mathrm{BR}$ 催化剂上存在晶格间距为 $0.37 \mathrm{~nm}$ 的纳米颗 粒，该晶面间距对应赤铁矿型 $\mathrm{Fe}_{2} \mathrm{O}_{3}$ 的(012)晶面; 催化 剂表面未发现明显金属 Pt 颗粒. 样品的 HRTEM 图表明 铁元素以赤铁矿形式存在于 $5 \mathrm{Fe} 1 \mathrm{Pt} \mathrm{BR}$ 催化剂, 这也与 粉末 X 射线衍射结果相印证. $5 \mathrm{Fe} 1 \mathrm{Pt}$ BR 催化剂的能量 色散 X 射线谱(EDS)结果显示了 $\mathrm{Fe}$ 和 $\mathrm{Pt}$ 在催化剂的分 布, 说明 $\mathrm{Fe}$ 和 $\mathrm{Pt}$ 的成功负载, $\mathrm{Pt}$ 元素更倾向于集中在铁 氧化物区域(图 3b).

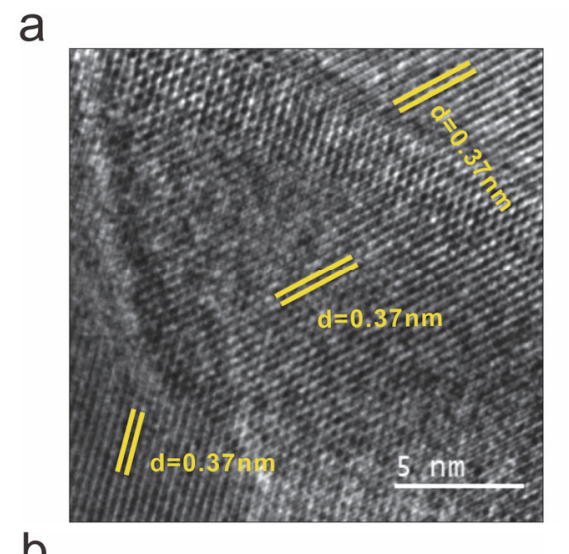

b

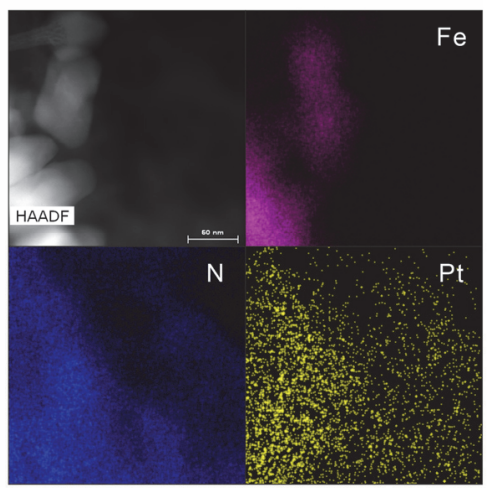

图 $35 \mathrm{Fe} 1 \mathrm{Pt}$ BR 的 HRTEM 图(a)和扫描透射电子显微镜-能量色散 X 射线谱(STEM-EDS)元素分布图(b)

Figure 3 HRTEM of 5Fe1Pt BR (a) and STEM-EDS elemental mapping of 5Fe1Pt BR (b)

5Fe1Pt AR 催化剂的高角环形暗场像(high angle annular dark-field imaging, HAADF)图(图 4a)中, 铁氧化 物颗粒为块体. 铁氧化物颗粒明亮处与颗粒周围(圆圈 标识处)均有Pt颗粒分布. STEM-EDS 元素分布图(图 S3) 显示 $5 \mathrm{Fe} 1 \mathrm{Pt} \mathrm{AR}$ 中 Pt 依然倾向于集中在铁氧化物颗粒表 面. 图 4b 中所示区域的 EDS 线扫也证明了线扫区域内 $\mathrm{Pt}$ 在铁氧化物表面较为均匀地分布. 这可能由于还原过 程中铂的迁移导致了还原后铁氧化物表面铂颗粒的产 生 ${ }^{[19]}$.
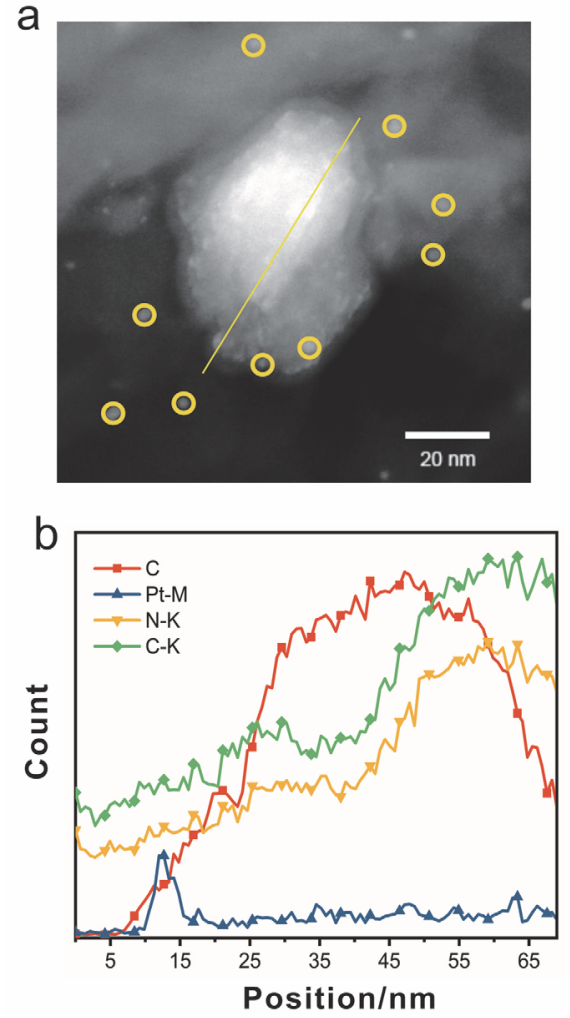

图 $45 \mathrm{Fe} 1 \mathrm{Pt} \mathrm{AR}$ 催化剂单个铁氧化物颗粒上的 EDS 线分析. EDS 分 析位置(a), 线扫图谱(b)

Figure 4 The position of the EDS line scan (a) and EDS line scan profile (b) of $\mathrm{FeO}_{x}$ particle on $5 \mathrm{Fe} 1 \mathrm{Pt} \mathrm{AR}$

如图 5a 所示, $5 \mathrm{Fe} 1 \mathrm{Pt} \mathrm{AR}$ 的 $\mathrm{N}_{2}$ 吸附-脱附等温线接 近 III 型等温线, 表明催化剂具有一定数量的大孔与介 孔. 计算得催化剂具有较大的比表面积 $\left(61.16 \mathrm{~m}^{2} / \mathrm{g}\right)$, 有 利于气体分子在催化剂表面的吸附; BJH 平均孔径为 $18.57 \mathrm{~nm}$, DFT 脱附孔径有层次地分布在微孔、介孔、 大孔范围(图 5b). 这种多级多孔结构被认为可以增强光 散射, 提高光催化效率, 而且大孔的引入可以减小扩散 阻力, 促进原料气和产物的分离与传质过程 ${ }^{[20]}$.

\section{2 活性}

我们使用图 S4 所示的反应器评价催化剂的活性, 无外加热源, 调节光强使反应温度保持在 $360{ }^{\circ} \mathrm{C}$, 具体 条件见实验部分, 活性测试结果如图 6a. 结果显示, $5 \mathrm{Fe} 1 \mathrm{Pt}$ AR 较对照组催化剂具备最佳的反应活性(7.36 $\left.\mathrm{mmol} \cdot \mathrm{h}^{-1} \cdot \mathrm{g}_{\mathrm{cat}}{ }^{-1}\right)$ 与高达 $97 \%$ 的一氧化碳选择性. 其中, $5 \mathrm{Fe} 0 \mathrm{Pt}$ 催化剂即使在最大光强下依然远未达到反应温 度 $\left(360{ }^{\circ} \mathrm{C}\right)$, 最高只能达到 $270{ }^{\circ} \mathrm{C}$, 此时气相色谱 FID 检测器中未检出反应产物, 故记其活性为 $0.0 \mathrm{Fe} 1 \mathrm{Pt}$, $0.5 \mathrm{Fe} 1 \mathrm{Pt}$ 催化剂活性分别为 0.54 和 $0.57 \mathrm{mmol} \cdot \mathrm{h}^{-1} \cdot \mathrm{g}_{\mathrm{cat}}{ }^{-1}$. 对比 $5 \mathrm{Fe} 1 \mathrm{Pt} \mathrm{AR}, 0 \mathrm{Fe} 1 \mathrm{Pt}, 0.5 \mathrm{Fe} 1 \mathrm{Pt}$ 催化剂的活性数据, 可知铁氧化物的加入显著增强了催化活性. 在长达 $30 \mathrm{~h}$ 的稳定性试验中, $5 \mathrm{Fe} 1 \mathrm{Pt}$ 催化剂表现出了优秀的稳定性 (图 S5). 


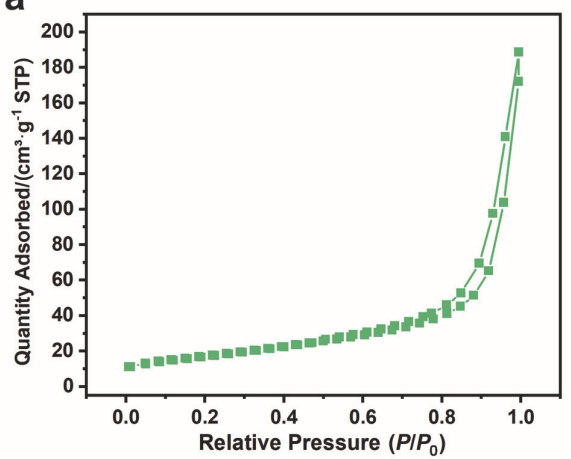

b

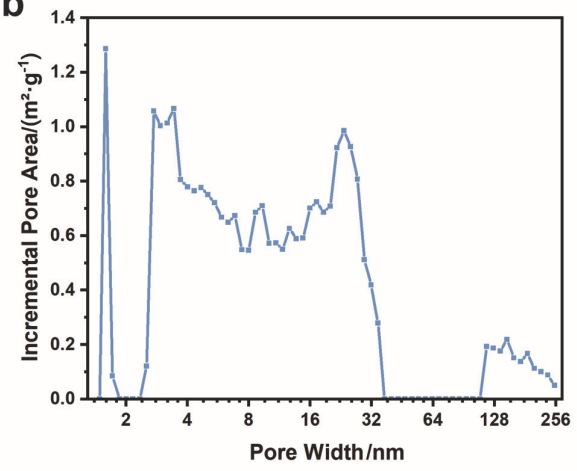

图 $55 \mathrm{Fe} 1 \mathrm{Pt} \mathrm{AR}$ 的 $\mathrm{N}_{2}$ 吸附-脱附等温线(a)和 DFT 脱附孔径分布增量 图(b)

Figure $5 \quad \mathrm{~N}_{2}$ absorption-desorption isotherm (a) and DFT desorption pore size distribution plot (b) of $5 \mathrm{Fe} 1 \mathrm{Pt} \mathrm{AR}$
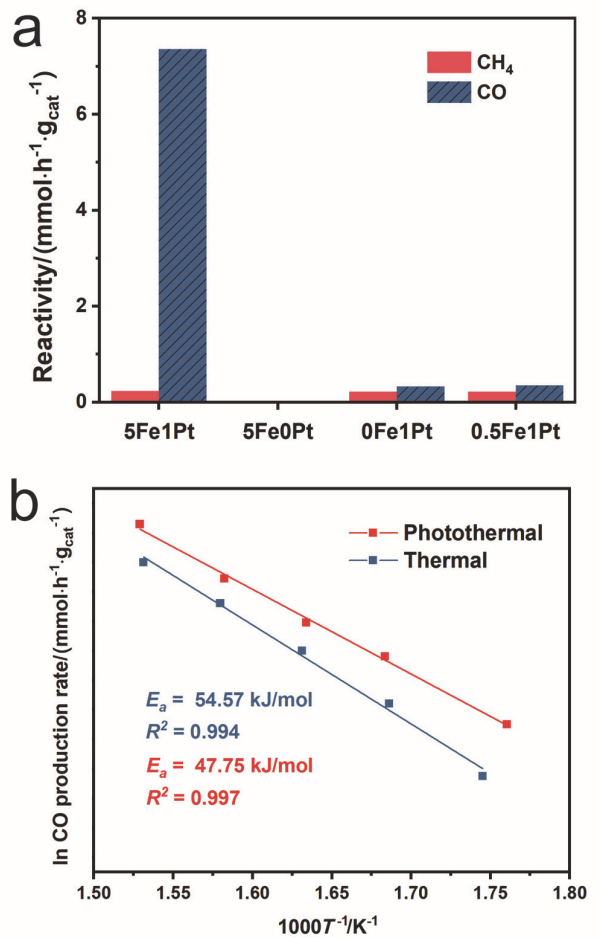

图 $65 \mathrm{Fe} 1 \mathrm{Pt} 、 5 \mathrm{Fe} 0 \mathrm{Pt} 、 0 \mathrm{Fe} 1 \mathrm{Pt}$ 和 $0.5 \mathrm{Fe} 1 \mathrm{Pt}$ 的甲烷/一氧化碳产生速率 (a)与光热-热表观活化能对比(b)

Figure $6 \mathrm{CH}_{4}$ and $\mathrm{CO}$ evolution rates over $5 \mathrm{Fe} 1 \mathrm{Pt}, 5 \mathrm{Fe} 0 \mathrm{Pt}, 0 \mathrm{Fe} 1 \mathrm{Pt}$, and $0.5 \mathrm{Fe} 1 \mathrm{Pt}(\mathrm{a})$, and apparent photothermal-thermal activation energy contrast (b)
调节光强改变光热反应温度，测得 $5 \mathrm{Fe} 1 \mathrm{Pt} \mathrm{AR}$ 在 300、320、340、360、380 ${ }^{\circ} \mathrm{C}$ 下的反应活性，对反应活 性的自然对数与反应温度的倒数作图(图 6b), 拟合得到 表观活化能为 $47.75 \mathrm{~kJ} / \mathrm{mol}$, 低于热反应条件下催化剂 测得表观活化能 $(54.57 \mathrm{~kJ} / \mathrm{mol})$, 说明催化反应过程中光 照对反应具有显著的促进作用.

在光功率为 $1700 \sim 3200 \mathrm{~mW} / \mathrm{cm}^{2}$ 范围内对催化剂 原位条件的光热转换效率进行了评价. 结果如图 7 所示, $5 \mathrm{Fe} 1 \mathrm{Pt}$ 较 $5 \mathrm{Fe} 0 \mathrm{Pt}$ 和 $0.5 \mathrm{Fe} 1 \mathrm{Pt}$ 在光照下可以产生更高的 温度，这表明 $5 \mathrm{Fe} 1 \mathrm{Pt}$ 具有更高的光热转化效率.

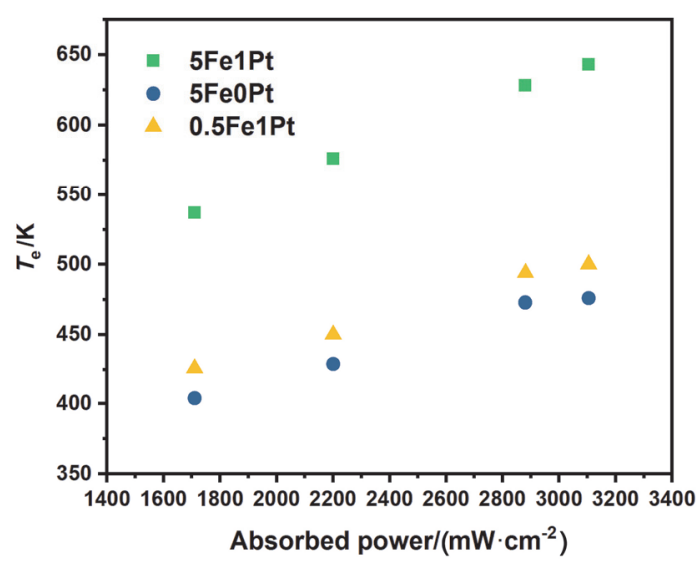

图 $75 \mathrm{Fe} 1 \mathrm{Pt} 、 5 \mathrm{Fe} 0 \mathrm{Pt}$ 和 $0.5 \mathrm{Fe} 1 \mathrm{Pt}$ 不同光强下的热平衡温度

Figure 7 Thermal equilibrium temperatures for $5 \mathrm{Fe} 1 \mathrm{Pt}, 5 \mathrm{Fe} 0 \mathrm{Pt}$ and $0.5 \mathrm{Fe} 1 \mathrm{Pt}$ at different power levels

总之, 铁氧化物与铂的引入增强了催化剂的光热转 化效率，显著降低了反应活化能，提高了催化剂光热催 化二氧化碳还原活性.

\section{3 反应机理}

通过使用紫外可见近红外漫反射光谱(DRS)、稳态 荧光光谱(PL)、光电流分析与原位傅里叶漫反射红外光 谱(DRIFTS), 我们对材料的光学性质和光热还原二氧 化碳反应机理进行了探究, 以解释 $5 \mathrm{Fe} 1 \mathrm{Pt}$ AR 催化剂较 对照组催化剂的高活性.

利用紫外可见分光光度计可以对比不同催化剂在 全太阳光波段对于光子的吸收能力, 结果如图 $8 \mathrm{a}$ 所示. 5Fe1Pt AR 催化剂较其他各组催化剂在全太阳光谱范围 $(250 \sim 2400 \mathrm{~nm})$ 均拥有更强的吸收，这源于 $\mathrm{Fe}_{3} \mathrm{O}_{4}$ 窄带 隙产生的宽光谱响应范围 ${ }^{[21]}$. 5Fe1Pt AR 在可见-近红外 波段可观的吸收强度意味着催化剂能够更好地利用红 外光，具有优异的光热转化性能.

对 $5 \mathrm{Fe} 1 \mathrm{Pt}$ AR 催化剂与 $\mathrm{g}-\mathrm{C}_{3} \mathrm{~N}_{4}$ 的吸收光谱数据运用 Kubelka-Munk 方程(式(1))进行转化:

$$
\alpha h v=A\left(h v-E_{\mathrm{g}}\right)^{n / 2}
$$

其中 $\alpha$ 为光吸收系数, $h$ 为普朗克常数, $v$ 为光波频率, $A$ 为与材料性质有关的常数, $E_{\mathrm{g}}$ 为样品的带隙宽度, 材料 为直接带隙半导体时 $n=1^{[22]}$. 使用直线外推法得到二 
者的禁带宽度分别为 2.78 与 $2.93 \mathrm{eV}$ (图 S6), 说明还原 后的铁氧化物使催化剂的禁带宽度减少了 $0.15 \mathrm{eV}$, 更 有利于光生载流子的产生. 与带隙宽度匹配的光子被用 于激发价带电子, 剩余的光能转化为热能, 实现全太阳 光谱范围(250 2400 nm) 的光能利用(图 6a). 高效的光 热转换产生的局域热场能有效促进吸附于催化剂表界 面的小分子进行交换, 大幅度降低反应分子在界面转移 的能垒 ${ }^{[23]}$.

图 $8 \mathrm{~b}$ 为 $330 \mathrm{~nm}$ 单色光激发下 $\mathrm{g}-\mathrm{C}_{3} \mathrm{~N}_{4} 、 5 \mathrm{Fe} 0 \mathrm{Pt}$ 、 $5 \mathrm{Fe} 1 \mathrm{Pt} \mathrm{AR}$ 的稳态苂光光谱. $\mathrm{g}-\mathrm{C}_{3} \mathrm{~N}_{4}$ 的苂光光谱在 $\lambda=$ $460 \mathrm{~nm}$ 处具有最高的荧光发射强度, $5 \mathrm{Fe} 0 \mathrm{Pt}$ 次之, $5 \mathrm{Fe} 1 \mathrm{Pt}$ AR 拥有最低的发射强度. $5 \mathrm{Fe} 1 \mathrm{Pt}$ AR 最低的荧光 强度说明 $5 \mathrm{Fe} 1 \mathrm{Pt} \mathrm{AR}$ 具有最低的载流子复合率. $5 \mathrm{Fe} 1 \mathrm{Pt}$ $\mathrm{AR}$ 光照下的低载流子复合率得益于高电导率的 $\mathrm{Fe}_{3} \mathrm{O}_{4}$ 通过两相界面处接触电场促进电子-空穴对的分离与转 移 ${ }^{[24]}$. 根据文献报道, $\mathrm{Fe}_{3} \mathrm{O}_{4}$ 的导带底 $(-5.73 \mathrm{eV}$ vs. 绝 对真空能级 AVS)、价带顶 $(-5.83 \mathrm{eV}$ vs. AVS)均在 $\mathrm{g}-\mathrm{C}_{3} \mathrm{~N}_{4}$ 禁带 $(-2.82 \sim-5.87 \mathrm{eV}$ vs. AVS $)$ 中, 二者之间会 形成I型异质结. g- $\mathrm{C}_{3} \mathrm{~N}_{4}$ 产生的光生电子与空穴均会迁 移、富集到 $\mathrm{Fe}_{3} \mathrm{O}_{4}$, 用于二氧化碳的光热还原反应 ${ }^{[24-27]}$.
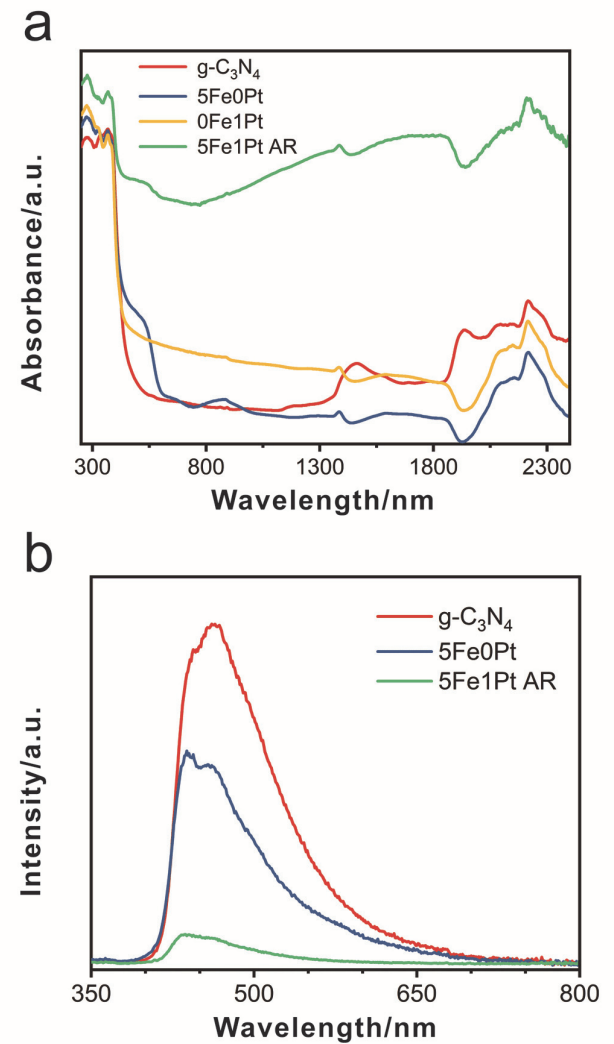

图 8 各组样品的 DRS (a)和荧光(b)谱图

Figure 8 The DRS spectra (a) and photoluminescence (PL) spectra (b) of samples

光电流分析是研究催化剂电荷分离和迁移的重要 手段. 我们使用光电流密度分析对 $5 \mathrm{Fe} 1 \mathrm{Pt} \mathrm{AR} 、 5 \mathrm{Fe} 0 \mathrm{Pt}$ 、 $\mathrm{g}-\mathrm{C}_{3} \mathrm{~N}_{4}$ 的光电性能进行了表征, 结果如图 9 所示. $5 \mathrm{Fe} 1 \mathrm{Pt}$
$\mathrm{AR}$ 在光照条件下具有最高的电流强度, 这表明 $5 \mathrm{Fe} 1 \mathrm{Pt}$ $\mathrm{AR}$ 较 $5 \mathrm{Fe} 0 \mathrm{Pt} 、 \mathrm{~g}-\mathrm{C}_{3} \mathrm{~N}_{4}$ 具有最高的光生载流子分离效率.

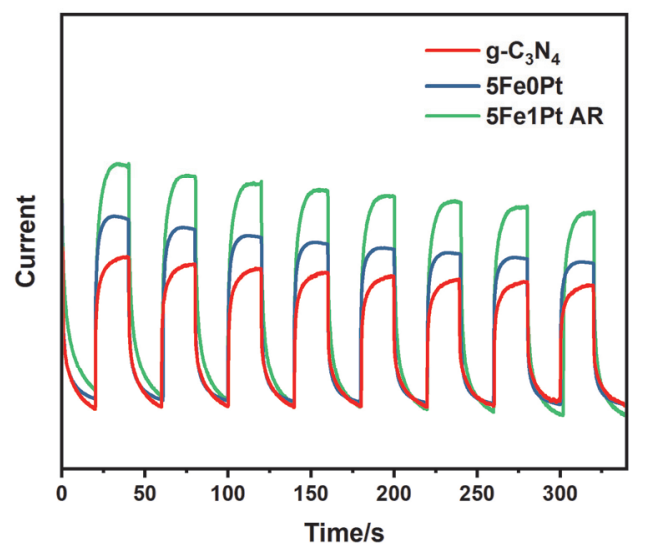

图 $95 \mathrm{Fe} 1 \mathrm{Pt} \mathrm{AR} 、 5 \mathrm{Fe} 0 \mathrm{Pt}$ 和 $\mathrm{g}-\mathrm{C}_{3} \mathrm{~N}_{4}$ 的光电流分析结果 Figure 9 Photocurrent of $5 \mathrm{Fe} 1 \mathrm{Pt}, 5 \mathrm{Fe} 0 \mathrm{Pt}$ and $\mathrm{g}-\mathrm{C}_{3} \mathrm{~N}_{4}$

为检测催化剂光热还原二氧化碳过程中的重要中 间体，我们使用原位傅里叶漫反射红外光谱(DRIFTS) 在纯氢气、 $\mathrm{CO}_{2} / \mathrm{H}_{2}(V / V, 1 / 1)$ 原料气、使用氢气吹扫二氧 化碳三组反应场景下对催化剂表面在 $370{ }^{\circ} \mathrm{C}$ 、光照条件 的反应历程进行了表征, 结果如图 10 所示.

(1) 纯氢气氛围.

将催化剂在氦气中加热至 $400{ }^{\circ} \mathrm{C}$ 去除表面吸附的 有机物和水, 恢复至 $370{ }^{\circ} \mathrm{C}$, 通入氢气. 图 10a 中于 $3620 \mathrm{~cm}^{-1}$ 处的圆形吸收峰归属于 $\mathrm{Fe}_{3} \mathrm{O}_{4}$ 表面羟基的吸 收峰, $3084 \mathrm{~cm}^{-1}$ 处吸附态水分子吸收的缺失表明该温度 下催化剂表面水分子已经完全脱附 ${ }^{[28]}$. 因此, $3620 \mathrm{~cm}^{-1}$ 处的羟基吸收源于铁氧化物表面在氢气氛围下的还原, 而不是水分子在铁氧化物表面的解离. 铁氧化物产生的 表面羟基可以显著增强二氧化碳在催化剂表面的吸附, 促进铁氧化物表面 $\mathrm{HCO}_{3}{ }^{-}$物种的产生 ${ }^{[29]}$.

(2) $\mathrm{CO}_{2} / \mathrm{H}_{2}(V / V, 1 / 1)$ 氛围.

向 $\mathrm{CO}_{2}$ 吸附饱和的体系中通入 $\mathrm{CO}_{2} / \mathrm{H}_{2}(V / V, 1 / 1)$ 合 成气. 结果如图 $10 \mathrm{~b} 、 10 \mathrm{c}$ 所示, 除气相中二氧化碳分子 于 2362、3510 3760 $\mathrm{cm}^{-1}$ 处的吸收峰外, 反应条件下 仅于 1076、1212、1739 $\mathrm{cm}^{-1}$ 处出现归属于 $\mathrm{CO}_{3}{ }^{2-} 、 \mathrm{HCO}_{3}{ }^{-}$ 物种的特征峰, 未发现 $\mathrm{COOH} 、 \mathrm{CO}_{2}{ }^{\delta} 、 \mathrm{CH}$ 等物种明显 的吸收峰, 说明反应条件下含碳物种在催化剂表面仅以 $\mathrm{CO}_{3}{ }^{2-}$ 和 $\mathrm{HCO}_{3}{ }^{-}$形式存在 ${ }^{[28-31]}$.

(3) 催化剂在氦气氛围加热至 $400{ }^{\circ} \mathrm{C}$ 后, 置于纯二 氧化碳气氛, 并向其中逐渐通入氢气至将二氧化碳赶 尽.

图 10d 中有两个弱峰发生了转变: 位于 $2076 \mathrm{~cm}^{-1}$, 归属于 $\mathrm{CO}$ 线性吸附在铂位点时碳氧键的伸缩振动吸收 峰, 与位于 $1999 \mathrm{~cm}^{-1}$, 归属于 CO 吸附在高度还原的铁 位点时碳氧键的伸缩振动吸收峰 ${ }^{[32-33]}$. 初始条件下, $2076 \mathrm{~cm}^{-1}$ 处的吸收源于此前还原过程中催化剂残留的 

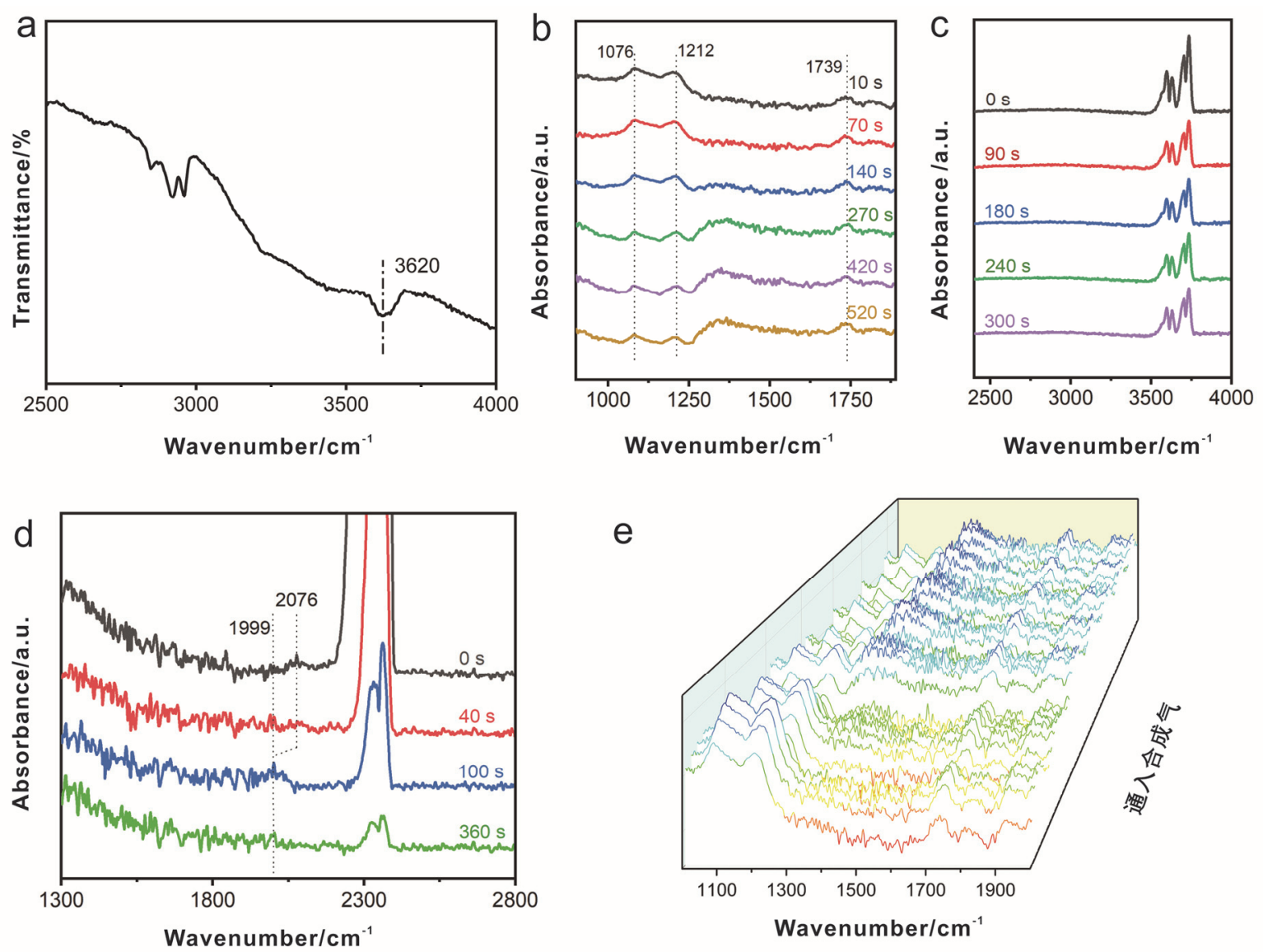

图 $105 \mathrm{Fe} 1 \mathrm{Pt} \mathrm{AR}$ 在 $\mathrm{H}_{2}$ 氛围中的红外吸收(a), 向 $\mathrm{CO}_{2}$ 处理后的 $5 \mathrm{Fe} 1 \mathrm{Pt} \mathrm{AR}$ 通入 $\mathrm{CO}_{2} / \mathrm{H}_{2}(\mathrm{~V} / \mathrm{V}, 1 / 1)$ 原料气后的原位 DRIFTS 变化 $(\mathrm{b}, \mathrm{c})$, 向 $\mathrm{CO}$ 处理 后的 $5 \mathrm{Fe} 1 \mathrm{Pt} \mathrm{AR}$ 通入 $\mathrm{H}_{2}$ 的原位 DRIFTS 变化(d), 不同气体条件吸收变化总谱(e)

Figure 10 IR spectra of $5 \mathrm{Fe} 1 \mathrm{Pt}$ AR under $\mathrm{H}_{2}$ (a). Operando DRIFTS for $5 \mathrm{Fe} 1 \mathrm{Pt}$ AR following exposure to $\mathrm{CO}_{2} / \mathrm{H}_{2}(V / V, 1 / 1)$ feed gas after pretreatment with pure $\mathrm{CO}_{2}(\mathrm{~b}, \mathrm{c})$, following exposure to $\mathrm{H}_{2}$ after pretreatment with pure $\mathrm{CO}_{2}(\mathrm{~d})$, and absorption changes under different reaction conditions (e)

表面还原物种在二氧化碳氛围中产生的微量 $\mathrm{CO}$ 在催化 剂表面的吸附. 通入氢气前, 二者仅存在 $2076 \mathrm{~cm}^{-1}$ 位 置的吸收; 通入氢气 $40 \mathrm{~s}$ 后, $2076 \mathrm{~cm}^{-1}$ 处峰强度减弱, $1999 \mathrm{~cm}^{-1}$ 处的峰出现并随氢气通入时间先增加后降低. 两组峰随氢气通入时间的变化说明, 通入氢气前, 产生 的 $\mathrm{CO}$ 仅吸附在铂上; 随着少量氢气的加入, 与氢气竞 争吸附在铂位点上的 $\mathrm{CO}$ 转移到了铁位点上; 持续通入 氢气, 催化剂吸附的 $\mathrm{CO}$ 均被吹扫出体系. 这意味着在 富含氢气的反应条件下, 铂并不会直接参与含碳物种的 转化过程. 因此反应过程中, 铂仅起到活化氢的作用, 铁氧化物是催化二氧化碳还原的活性位点.

在向二氧化碳氛围逐渐通入合成气的过程中, 我们 发现催化剂在 $4000 \sim 1000 \mathrm{~cm}^{-1}$ 波段的吸收强度与氢气 组分占比呈正相关, 这种差异在 $1240 \sim 1900 \mathrm{~cm}^{-1}$ 波段 尤为强烈 (图 10e). 与之相似地, 贵金属/二氧化钛等体 系在氢气条件下也会在红外/近红外区出现宽的吸收增 强现象. 在贵金属/二氧化钛体系中, 这种吸收增强被认 为是氢溢流现象发生的标志: 氢气在贵金属位点被解离 成氢原子, 氢原子溢流至二氧化钛的氧原子, 形成 $\mathrm{Ti}-\mathrm{O}(\mathrm{H})-\mathrm{Ti}$ 物种, 氢原子携带的电子注入能量低于导带
$0.12 \mathrm{eV}$ 的浅缺陷态，使材料 “金属化”，进而可以被红 外光激发到导带, 引起宽的红外吸收 ${ }^{[34-35]}$. 与二氧化钛 中带隙来源为电子从 $\mathrm{O}^{2-}$ 到 $\mathrm{Ti}^{4+}$ 的跃迁不同, $\mathrm{Fe}_{3} \mathrm{O}_{4}$ 带隙 主要来源于八面体位置 $\mathrm{Fe}^{2+}$ 与 $\mathrm{Fe}^{3+}$ 之间通过小极化子 跃迁的电荷转移 ${ }^{36]}$. 反应气氛下, 吸附氢的 $\mathrm{Fe}_{3} \mathrm{O}_{4}$ 表面 发生氢化还原, $\mathrm{Fe}^{3+}$ 部分转变为 $\mathrm{Fe}^{2+}$, 带隙逐渐消失, 表 面发生半导体-半金属转变, 从而也可以引起与贵金属/ 二氧化钛体系氢溢流现象类似的红外吸收 ${ }^{[37-39]}$. 因此, 本体系出现的吸收增强现象说明反应条件下 $5 \mathrm{Fe} 1 \mathrm{Pt} \mathrm{AR}$ 催化剂表面存在氢溢流.

根据 DRIFTS 结果, 我们提出了 $5 \mathrm{Fe} 1 \mathrm{Pt}$ AR 催化剂 光热还原二氧化碳的可能的反应机理. 如图 11 所示, 反 应机理包括三步: (1)二氧化碳在表面羟基协助下吸附到 铁氧化物表面, 快速形成碳酸盐/碳酸氢盐 ${ }^{[40] ;}$ (2)碳酸 盐/碳酸氢盐在铁氧化物表面脱氧分解生成一氧化碳 ${ }^{[41]}$; (3)活性位点加氢还原、再生.

\section{3 结论}

光热催化可以通过光物理层面的光-热转化, 驱动 催化剂表面的化学反应, 直接将光能转化为化学能. 本 


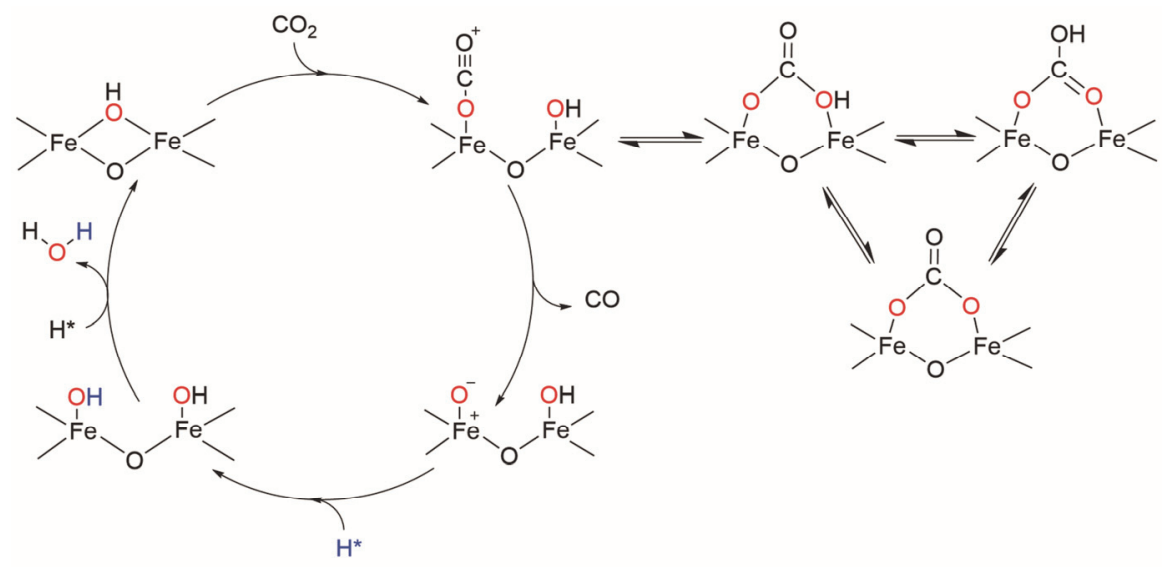

图 11 可能的光热 $\mathrm{CO}_{2}$ 还原机理示意图

Figure 11 Illustration of possible mechanism of photothermal $\mathrm{CO}_{2}$ reduction

工作报道了一种通过水热、浸渍两步法合成的石墨相氮 化碳催化剂, 催化剂在光热 $360{ }^{\circ} \mathrm{C}$ 反应条件下实现了 $7.36 \mathrm{mmol} \cdot \mathrm{h}^{-1} \cdot \mathrm{g}_{\mathrm{cat}}{ }^{-1}$ 的二氧化碳还原活性与 $97 \%$ 的一氧 化碳选择性. 结合 DRS、PL、光电流分析和原位 DRIFTS 表征结果, 我们证明了铂与铁氧化物的引入提供了氢 气、二氧化碳反应位点, 增强了载流子分离效率与全谱 太阳光吸收能力, 显著提升了催化剂的光热转换效率. 基于原位 DRIFTS 结果, 我们提出了催化历程中含碳物 种在铁氧化物表面转化的可能的反应机理, 并证明了铂 在 $\mathrm{Fe}_{3} \mathrm{O}_{4}$ 表面的氢溢流效应. 本工作中 $\mathrm{g}-\mathrm{C}_{3} \mathrm{~N}_{4}$ 的改性策 略与对 $\mathrm{CO}_{2}$ 还原的机理讨论也将为今后开发高效的二 氧化碳光热还原催化剂提供一定的参考价值.

\section{4 实验部分}

\section{$4.1 \mathrm{~g}-\mathrm{C}_{3} \mathrm{~N}_{4}$ 的合成}

称取 $10 \mathrm{~g}$ 尿素 $\left(\mathrm{CH}_{4} \mathrm{~N}_{2} \mathrm{O}\right.$, 阿拉丁, $\left.\mathrm{AR}\right)$ 于刚玉坩埚, 使用马弗炉以 $5{ }^{\circ} \mathrm{C} / \mathrm{min}$ 的速率升温至 $550{ }^{\circ} \mathrm{C}$, 在空气 中灼烧 $4 \mathrm{~h}$. 坩埚中留存的淡黄色粉末在磁力搅拌的情 况下投入到 $0.1 \mathrm{~mol} / \mathrm{L}$ 硝酸溶液 $\left(\mathrm{HNO}_{3}\right.$, 沪试)中, 持续 搅拌 $8 \mathrm{~h}$. 产物经过去离子水、无水乙醇多次洗涤、离心 后, 放入 $50{ }^{\circ} \mathrm{C}$ 真空干燥箱干燥过夜.

\section{$4.2 x F e 0 P t$ 的合成}

称取 $250 \mathrm{mg}$ 前一步制备的 $\mathrm{g}-\mathrm{C}_{3} \mathrm{~N}_{4}$ 于配备聚四氟乙 烯内衬的不锈钢反应釜中, 加入 $30 \mathrm{~mL}$ 去离子水, 搅拌 至均匀. 称取适量的 $\mathrm{FeCl}_{3} \cdot 6 \mathrm{H}_{2} \mathrm{O}$ (阿拉丁, $\mathrm{ACS}$ ), 加入到 混合液体中, 搅拌 $20 \mathrm{~min}$. 滴入 $0.5 \mathrm{~mL}$ 饱和氨水(沪试, $\mathrm{AR}$ ), 继续搅拌 $20 \mathrm{~min}$. 搅拌完成后, 将组装好的反应 釜置于烘箱中, 加热 $150{ }^{\circ} \mathrm{C}$ 保持 $5 \mathrm{~h}$. 产物经过去离子 水、无水乙醇多次洗涤、离心后, 放入 $50{ }^{\circ} \mathrm{C}$ 真空干燥 箱干燥过夜.

\section{$4.3 x F e 1 P t$ 的合成}

称取 $200 \mathrm{mg} \times \mathrm{Fe} 0 \mathrm{Pt}$ 或 $\mathrm{g}-\mathrm{C}_{3} \mathrm{~N}_{4}$ 于研钵中, 根据负载
量加入适量事先配制的 $\mathrm{H}_{2} \mathrm{PtCl}_{6} \cdot 6 \mathrm{H}_{2} \mathrm{O}$ (阿拉丁, ACS)乙 醇溶液, 研磨成均一浆液后使用红外灯烘干剩余乙醇. 获得固体转移至刚玉瓷舟中, 使用管式炉以 $5{ }^{\circ} \mathrm{C} / \mathrm{min}$ 升温至 $270{ }^{\circ} \mathrm{C}$, 于氮气氛中加热 $2 \mathrm{~h}$.

\section{4 材料表征}

采用 X-射线粉末衍射仪(D8 Advance)、球差校正扫 描透射电镜(FEI Titan ${ }^{\mathrm{TM}} \mathrm{G}^{2}$ 60-300)、 $X$ 射线光电子能谱 (Thermo Fisher Scientific K-Alpha spectrometer)表征样 品的形貌和成分. 材料光学性能测试由岛津 UV-3600 (DRS)、爱丁堡 FLS1000 (PL)完成. 原位漫反射红外傅 里叶变换光谱数据由 BRUKER 80v 采集, 反应温度保持 在 $370{ }^{\circ} \mathrm{C}$. 比表面积与孔径信息由麦克 ASAP2460 全自 动比表面及孔隙度分析仪获得. 光电流分析使用 CHI660E 电化学工作站, 于铁氰化钾溶液中在 $0.2 \mathrm{~V}$ 开 路电压条件下进行测定. 光功率由 CEL-NP2000-2A 全 自动光功率计获得.

\section{5 活性测试}

本实验使用 $300 \mathrm{~W}$ 氙灯 (中教金源, CEL-HXF300 光 催化氙灯光源)作为光源, 热电偶作为温度指示器, 无 额外热源, 反应原料气中 $\mathrm{CO}_{2}$ 和 $\mathrm{H}_{2}$ 体积比为 $1: 1$, 流 速均为 $5 \mathrm{~mL} / \mathrm{min}$, 气体流速由气体流量计确定, 气体组 分由气相色谱(Agilent 7890B GC, 配备 FID 检测器) 定 量测定, 以峰面积为定量依据. 称取 $50 \mathrm{mg} 5 \mathrm{Fe} 1 \mathrm{Pt} \mathrm{AR}$, $5 \mathrm{Fe} 0 \mathrm{Pt}, 0 \mathrm{Fe} 1 \mathrm{Pt}, 0.5 \mathrm{Fe} 1 \mathrm{Pt}$ 催化剂投入石英反应器中, 调 节光强使反应温度维持在 $360{ }^{\circ} \mathrm{C}$, 测得反应活性. 热对 比实验使用类似条件在中教金源 CEL-GPPCM 微型光 热催化微反系统装置中进行.

\section{References}

[1] Gao, W.; Liang, S.; Wang, R.; Jiang, Q.; Zhang, Y.; Zheng, Q.; Xie, B.; Toe, C. Y.; Zhu, X.; Wang, J.; Huang, L.; Gao, Y.; Wang, Z.; Jo, C.; Wang, Q.; Wang, L.; Liu, Y.; Louis, B.; Scott, J.; Roger, A.-C.; Amal, R.; He, H.; Park, S.-E. Chem. Soc. Rev. 2020, 49, 8584.

[2] Ghoussoub, M.; Xia, M.; Duchesne, P. N.; Segal, D.; Ozin, G. Energy Environ. Sci. 2019, 12, 1122. 
[3] Yin, S.; Sun, L.; Zhou, Y.; Li, X.; Li, J.; Song, X.; Huo, P.; Wang, H.; Yan, Y. Chem. Eng. J. 2021, 406, 126776.

[4] Zhang, X.; Deng, B.; Fan, H.; Huang, W.; Zhang, Y. Acta Chim. Sinica. 2020, 78, 1120 (in Chinese). (张旭寒, 邓博文, 范海东, 黄 文辉, 张彦威, 化学学报, 2020, 78, 1120.)

[5] Hoch, L. B.; O’Brien, P. G.; Jelle, A.; Sandhel, A.; Perovic, D. D.; Mims, C. A.; Ozin, G. A. ACS Nano 2016, 10, 9017.

[6] Wang, L.; Dong, Y.; Yan, T.; Hu, Z.; Jelle, A. A.; Meira, D. M.; Duchesne, P. N.; Loh, J. Y. Y.; Qiu, C.; Storey, E. E.; Xu, Y.; Sun, W.; Ghoussoub, M.; Kherani, N. P.; Helmy, A. S.; Ozin, G. A. Nat. Commun. 2020, 11, 2432.

[7] Hoch, L. B.; O’Brien, P. G.; Jelle, A.; Sandhel, A.; Perovic, D. D.; Mims, C. A.; Ozin, G. A. ACS Nano 2016, 10, 9017.

[8] Li, Y. F.; Lu, W.; Chen, K.; Duchesne, P.; Jelle, A.; Xia, M.; Wood, T. E.; Ulmer, U.; Ozin, G. A. J. Am. Chem. Soc. 2019, 141, 14991

[9] Jia, J.; Wang, H.; Lu, Z.; O’Brien, P. G.; Ghoussoub, M.; Duchesne, P.; Zheng, Z.; Li, P.; Qiao, Q.; Wang, L.; Gu, A.; Jelle, A. A.; Dong, Y.; Wang, Q.; Ghuman, K. K.; Wood, T.; Qian, C.; Shao, Y.; Qiu, C.; Ye, M.; Zhu, Y.; Lu, Z. H.; Zhang, P.; Helmy, A. S.; Singh, C. V.; Kherani, N. P.; Perovic, D. D.; Ozin, G. A. Adv. Sci. 2017, 4, 1700252.

[10] Li, C.; Chen, F.; Ye, L.; Li, W.; Yu, H.; Zhao, T. Acta Chim. Sinica 2020, 78, 1448 (in Chinese). (李宸, 陈凤华, 叶丽, 李伟, 于晗, 赵栦, 化学学报, 2020, 78, 1448.)

[11] Wang, X.; Maeda, K.; Thomas, A.; Takanabe, K.; Xin, G.; Carlsson, J. M.; Domen, K.; Antonietti, M. A. Nat. Mater. 2009, 8, 76.

[12] Ong, W. J.; Tan, L. L.; Ng, Y. H.; Yong, S. T.; Chai, S. P. Chem. Rev. 2016, 116,7159 .

[13] Huang, Z. F.; Song, J.; Pan, L.; Wang, Z.; Zhang, X.; Zou, J. J.; Mi, W.; Zhang, X.; Wang, L. Nano Energy 2015, 12, 646.

[14] Chen, P.; Lei, B.; Dong, X.; Wang, H.; Sheng, J.; Cui, W.; Li, J.; Sun, Y.; Wang, Z.; Dong, F. ACS Nano 2020, 14, 15841.

[15] Chen, S.; Hu, Y.; Meng, S.; Fu, X. Appl. Catal., B 2014, 150-151, 564.

[16] Grosvenor, A. P.; Kobe, B. A.; Biesinger, M. C.; McIntyre, N. S. Surf. Interface Anal. 2004, 36, 1564.

[17] Palmer, J. W.; Swartz Jr, W. E.; King, D.; Stanko, J. A. Fla. Sci. 1993, 56, 123.

[18] Romeo, M.; Majerus, J.; Legare, P.; Castellani, N. J.; Leroy, D. B. Surf. Sci. 1990, 238, 163.

[19] Liu, H. Y.; Chiou, W. A.; Fröhlich, G.; Sachtler, W. M. H. Top. Catal. 2000, 10, 49 .

[20] Sun, M. H.; Huang, S. Z.; Chen, L. H.; Li, Y.; Yang, X. Y.; Yuan, Z. Y.; Su, B. L. Chem. Soc. Rev. 2016, 45, 3479.

[21] He, S.; Li, W.; Wang, X.; Ma, Q.; Li, M.; Xu, W.; Wang, X.; Zhao, C. Appl. Surf. Sci. 2020, 506, 144948 .
[22] Kong, T.; Stolze, K.; Timmons, E. I.; Tao, J.; Ni, D.; Guo, S.; Yang, Z.; Prozorov, R.; Cava, R. J. Adv. Mater. 2019, 31, 1.

[23] Wang, L.; Ghoussoub, M.; Wang, H.; Shao, Y.; Sun, W.; Tountas, A. A.; Wood, T. E.; Li, H.; Loh, J. Y. Y.; Dong, Y.; Xia, M.; Li, Y.; Wang, S.; Jia, J.; Qiu, C.; Qian, C.; Kherani, N. P.; He, L.; Zhang, X.; Ozin, G. A. Joule 2018, 2, 1369.

[24] Zhu, Z.; Lu, Z.; Wang, D.; Tang, X.; Yan, Y.; Shi, W.; Wang, Y.; Gao, N.; Yao, X.; Dong, H. Appl. Catal., B 2016, 182, 115.

[25] Xu, Y.; Schoonen, M. A. A. Am. Mineral. 2000, 543.

[26] Wu, H. Z.; Liu, L. M.; Zhao, S. J. Phys. Chem. Chem. Phys. 2014, $16,3299$.

[27] Moniz, S. J. A.; Shevlin, S. A.; Martin, D. J.; Guo, Z. X.; Tang, J. Energy Environ. Sci. 2015, 8, 731.

[28] Arndt, B.; Creutzburg, M.; Grånäs, E.; Volkov, S.; Krausert, K.; Vlad, A.; Noei, H.; Stierle, A. J. Phys. Chem. C 2019, 123, 26662.

[29] Mirabella, F.; Zaki, E.; Ivars-Barcelo, F.; Schauermann, S.; Shaikhutdinov, S.; Freund, H. J. J. Phys. Chem. C 2018, 122, 27433.

[30] Graciani, J.; Mudiyanselage, K.; Xu, F.; Baber, A. E.; Evans, J.; Senanayake, S. D.; Stacchiola, D. J.; Liu, P.; Hrbek, J.; Fernández Sanz, J.; Rodriguez, J. A. Science 2014, 345, 546.

[31] Hakim, A.; Marliza, T. S.; Abu Tahari, N. M.; Wan Isahak, R. W. N.; Yusop, R. M.; Mohamed Hisham, W. M.; Yarmo, A. M. Ind. Eng. Chem. Res. 2016, 55, 7888

[32] Gracia, F. J.; Bollmann, L.; Wolf, E. E.; Miller, J. T.; Kropf, A. J. J. Catal. 2003, 220, 382.

[33] Benziger, J. B.; Larson, L. R. J. Catal. 1982, 77, 550.

[34] Li, X.; Lin, J.; Li, L.; Huang, Y.; Pan, X.; Collins, S. E.; Ren, Y.; Su, Y.; Kang, L.; Liu, X.; Zhou, Y.; Wang, H.; Wang, A.; Qiao, B.; Wang, X.; Zhang, T. Angew. Chem. Int. Ed. 2020, 59, 2.

[35] Panayotov, D. A.; Yates, J. T. J. Phys. Chem. C 2007, 111, 2959.

[36] Skomurski, F. N.; Kerisit, S.; Rosso, K. M. Geochim. Cosmochim. Acta 2010, 74, 4234.

[37] Parkinson, G. S.; Mulakaluri, N.; Losovyj, Y.; Jacobson, P.; Pentcheva, R.; Diebold, U. Phys. Rev. B: Condens. Matter Mater. Phys. 2010, $82,125413$.

[38] Novotny, Z.; Mulakaluri, N.; Edes, Z.; Schmid, M.; Pentcheva, R.; Diebold, U.; Parkinson, G. S. Phys. Rev. B: Condens. Matter Mater. Phys. 2013, 87, 195410.

[39] Kurahashi, M.; Sun, X.; Yamauchi, Y. Phys. Rev. B: Condens. Matter Mater. Phys. 2010, 81, 193402.

[40] Baltrusaitis, J.; Jensen, J. H.; Grassian, V. H. J. Phys. Chem. B 2006, 110, 12005.

[41] Gálvez, M. E.; Loutzenhiser, P. G.; Hischier, I.; Steinfeld, A. Energy Fuels 2008, 22, 3544.

(Cheng, B.) 